\title{
Spatial Correlation in Quantum Chaotic Systems with Time-reversal Symmetry: Theory and Experiment
}

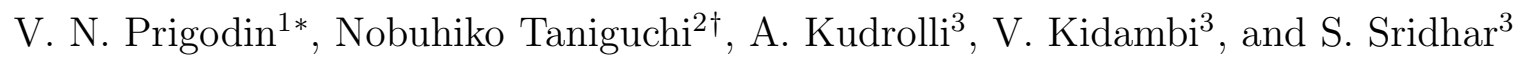 \\ ${ }^{1}$ Max-Planck-Institute für Physik komplexer Systeme, Außenstelle Stuttgart, Heisenbergstr.1, \\ 70569 Stuttgart, Germany \\ ${ }^{2}$ Department of Physics, Massachusetts Institute of Technology, Cambridge, MA 02139 \\ ${ }^{3}$ Department of Physics, Northeastern University, Boston, MA 02115
}

\begin{abstract}
The correlation between the values of wavefunctions at two different spatial points is examined for chaotic systems with time-reversal symmetry. Employing a supermatrix method, we find that there exist long-range Friedel oscillations of the wave function density for a given eigenstate, although the background wavefunction density fluctuates strongly. We show that for large fluctuations, once the value of the wave function at one point is known, its spatial dependence becomes highly predictable for increasingly large space around this point. These results are compared with the experimental wave functions obtained from billiard-shaped microwave cavities and very good agreement is demonstrated.
\end{abstract}

PACS numbers: 73.20.Dx, 73.20.Fz, 05.45.+b 
Quantum properties of classically chaotic systems such as "billiards" and quantum dots are revealed to have remarkable universal behaviors which depend only on the generic symmetry of the system, such as the time-reversal symmetry and/or the spin rotational symmetry [1 [ [ [ : It has been shown that the spectral statistics are well described by universal statistical correlations derived from the random matrix theory [4:5]. (See also [6 8] for reviews.) Complementary and comprehensive information beyond the energy statistics can be obtained by examining the statistics of chaotic wavefunctions. For example, the distribution of the local density in a fully chaotic system is known to be universal and obey the Porter-Thomas distribution [1, 9], which is given for a system with time-reversal symmetry, by the equation

$$
P_{0}(v) \equiv\left\langle\delta\left(v-V\left|\psi_{\epsilon}(\mathbf{r})\right|^{2}\right)\right\rangle=\frac{1}{\sqrt{2 \pi v}} \exp (-v / 2),
$$

where $\psi_{\epsilon}(\mathbf{r})$ is the eigenfunction with energy $\epsilon$ in a system with volume $V .\langle\cdots\rangle$ means the average over the disorder and/or irregular potential. Eq. (四) tells us that wavefunctions fluctuate strongly but in a universal way.

To get further understanding about the nature of chaotic wavefunctions, other statistical quantities which can characterize their spatial correlations are desirable and needed. The average behavior of the amplitude of the wavefunction has been conjectured by Berry to be similar to a pattern generated from random super-position of plane waves [10,11. Based on this assumption the average amplitude correlations were shown to be a Bessel function. Recently, these expressions have been derived within the supersymmetry formalism [12]. In this paper, taking a chaotic system with the time-reversal symmetry, correlations about a particular value of the wavefunction have been derived analytically and compared to experiments for the first time. This is not only a more stringent test of the universality of chaotic wavefunctions, but also gives us a handle on knowing the behavior of a wavefunction of the system, once the wavefunction is known only at a limited number of points. Our main object is to investigate the joint probability distribution function of the density for two different spatial points $\left(r=\left|\mathbf{r}_{1}-\mathbf{r}_{2}\right|\right)$ defined by 


$$
P\left(v_{1}, v_{2} ; r\right)=\left\langle\delta\left(v_{1}-V\left|\psi_{\epsilon}\left(\mathbf{r}_{1}\right)\right|^{2}\right) \delta\left(v_{2}-V\left|\psi_{\epsilon}\left(\mathbf{r}_{2}\right)\right|^{2}\right)\right\rangle .
$$

Although the relevant universality class for experiments which can directly observe the amplitude of wavefunctions, such as quantum corrals [13,14 and microwave cavity [15,16] is orthogonal, only the expression for $P\left(v_{1}, v_{2}\right)$ in the unitary case is known so far [17], because of technical difficulties. Here we evaluate $P\left(v_{1}, v_{2} ; r\right)$ for the orthogonal case by finding special techniques (Eq. (20) below). In the microwave cavity, the electromagnetic field obeys the same equation of motion as a quantum particle in a two-dimensional billiard. This enables us to make direct comparison between the analytical results and the experimental data. The experimental data for the wave function density was obtained from thin cylindrical microwave cavities of the Sinai stadium, by using a cavity perturbation technique [16], 18]. The wavefunction density data was earlier seen to be consistent with Eq. (11) [18], and also in agreement with the expression for density auto-correlations obtained in ref. [12]. Once we know $P\left(v_{1}, v_{2} ; r\right)$, we can also find the conditional probability

$$
P_{v_{1}}\left(v_{2} ; r\right)=P\left(v_{1}, v_{2} ; r\right) / P_{0}\left(v_{1}\right) \text {. }
$$

$P_{v_{1}}\left(v_{2} ; r\right)$ describes the distribution of the wave function of $v_{2}=V\left|\psi_{\epsilon}\left(\mathbf{r}_{2}\right)\right|^{2}$ at the point $\mathbf{r}_{2}$, provided that $v_{1}=V\left|\psi_{\epsilon}\left(\mathbf{r}_{1}\right)\right|^{2}$ at $\mathbf{r}_{1}$. Here we compare the coordinate dependence of the first and the second moments of Eq. (3) between theory and experiment.

The system under consideration can be expressed by the Hamiltonian

$$
H=\frac{1}{2 m} \mathbf{p}^{2}+U_{0}(\mathbf{r})+U_{1}(\mathbf{r}),
$$

where $U_{0}(\mathbf{r})$ denotes the regular part of a confining potential, and $U_{1}(\mathbf{r})$ is a random potential which is responsible for the chaotic dynamics - impurities or 'imperfection' of the shape of the system. We take the ensemble average over $U_{1}(\mathbf{r})$ by use of the supermatrix method, which reproduces the spectral correlations of Wigner-Dyson statistics [19,20] and recently was successfully applied to calculate other universal properties relating to chaotic wavefunctions [12,17,21,22]. 
We should remark that although the supermatrix method was originally derived from the Gaussian random potential where the mean free path $\ell$ is much smaller than the size of the system $L$, the ergodicity hypothesis [24] allows us to extend our present result for cases $\ell \sim L$ by identifying the averaging over space and different states for a given sample with that over disorder. As a confirmation of the ergodicity hypothesis we will demonstrate that the theoretical dependencies for wavefunctions derived from a disordered system with $\ell \ll L$ are universal and describe very well experimental results for quantum billiard systems for which $\ell \sim L$.

After lengthy calculations which we will sketch later, we have obtained the following analytical expression for $P\left(v_{1}, v_{2} ; r\right)$ for the orthogonal case:

$$
\begin{gathered}
P\left(v_{1}, v_{2} ; r\right)=\frac{1}{2 \pi f(r)} \int_{0}^{f(r)} \frac{p d p}{\sqrt{f^{2}(r)-p^{2}}}\left(1+p \frac{d}{d p}\right) \sqrt{\frac{1-p^{2}}{2 \pi v_{1} v_{2}}} \int_{0}^{\infty} \frac{d z}{\sqrt{z}} e^{z / 2} \varphi\left(\frac{v_{1}+z}{2}, \frac{v_{2}+z}{2} ; p\right) \\
\varphi\left(v_{1}, v_{2} ; p\right)=\frac{1}{1-p^{2}} I_{0}\left(\frac{2 p \sqrt{v_{1} v_{2}}}{1-p^{2}}\right) \exp \left(-\frac{v_{1}+v_{2}}{1-p^{2}}\right)
\end{gathered}
$$

where $I_{0}(p)$ is the modified Bessel function and $f(r)$ is the Friedel function [23]. Note that $P\left(v_{1}, v_{2} ; r\right)$ depends on $f(r)$ in an universal way and all coordinate dependence of $P\left(v_{1}, v_{2} ; r\right)$ is incorporated only through $f(r)$. In fact, the function $f(r)$ represents the average correlation of the amplitude of wave function [10,11]: $f(r)=V^{2}<\psi_{\epsilon}^{*}\left(r_{1}\right) \psi_{\epsilon}\left(r_{2}\right)>$. For the case of a flat background potential in a $d$-dimensional system, $f(r)$ becomes

$$
f(r)=\Gamma(d / 2)(2 / k r)^{d / 2-1} J_{d / 2-1}(k r) e^{-r / 2 \ell},
$$

where $k$ is the wave vector $\left(\epsilon=\hbar^{2} k^{2} / 2 m\right), J_{n}(x)$ is the Bessel function, and $\Gamma(n)$ is the gamma function. Note that the envelope of $f(r)$ decays like $(k r)^{-(d-1) / 2}$ for $k^{-1} \lesssim r \lesssim \ell$ and this behavior corresponds to the representation of chaotic wave function as a random superposition of plain waves [10,11].

It should be remarked that the same distribution function in the unitary case is given by $P\left(v_{1}, v_{2} ; r\right)=\varphi\left(v_{1}, v_{2} ; f(r)\right)$ [17. Due to two additional integrations in Eq. (5) the 
spatial correlations for the orthogonal case are weaker and the fluctuations are stronger in comparison with the unitary one.

We first check that $P\left(v_{1}, v_{2} ; r\right)$ given by Eq. (5), yields correct limiting behaviors. For remotely separate points such that $f(r) \approx 0$ the fluctuations of the wavefunction density within the given eigenstate become independent, i.e., $P\left(v_{1}, v_{2} ; r\right) \approx P_{0}\left(v_{1}\right) P_{0}\left(v_{2}\right)$. In the opposite limit of close enough points that $f(r) \approx 1$, there is an obvious strong correlation between fluctuations as

$$
P\left(v_{1}, v_{2} ; r\right) \approx \frac{P_{0}\left(v_{1}\right)}{\sqrt{8 \pi v_{1}\left(1-f^{2}\right)}} \exp \left[-\frac{\left(v_{1}-v_{2}\right)^{2}}{8 v_{1}\left(1-f^{2}\right)}\right] .
$$

From Eq. (8), we can extract information about the gradient of the wavefunction. By setting $\psi\left(\mathbf{r}_{2}\right)=\psi\left(\mathbf{r}_{1}\right)+r \nabla_{\mathbf{n}} \psi\left(\mathbf{r}_{1}\right)+O\left(r^{2}\right)$, and expanding for small $r$, we obtain the joint distribution involving the wavefunction and its gradient in any direction $\mathbf{n}=\mathbf{r} / r$. Accordingly we find that the gradient of the wavefunction along any direction fluctuates independently of the value of wavefunction, and obeys also the Porter-Thomas distribution:

$$
\left\langle\delta\left(v-V|\psi(\mathbf{r})|^{2}\right) \delta\left(s-\frac{V d}{2 k^{2}}\left|\nabla_{\mathbf{n}} \psi(\mathbf{r})\right|^{2}\right)\right\rangle=P_{0}(v) P_{0}(s) .
$$

This conclusion is, however, not true for higher gradients of the wavefunction.

The conditional probability $P_{v_{1}}\left(v_{2} ; r\right)$ is obtained straightforwardly with Eqs. (3,5). To see how the fluctuations of the wavefunctions behave and to compare between the analytical results and the experiments, the conditional average $\left\langle v_{2}\right\rangle_{v_{1}}$ and the conditional variance is more convenient. Denoting $\delta v_{2}=v_{2}-\left\langle v_{2}\right\rangle_{v 1}$, we obtain:

$$
\begin{aligned}
\left\langle v_{2}\right\rangle_{v_{1}} & =1+f^{2}(r)\left(v_{1}-1\right), \\
\left\langle\left(\delta v_{2}\right)^{2}\right\rangle_{v_{1}} & =2+4 f^{2}(r)\left(v_{1}-1\right)+2 f^{4}(r)\left(1-2 v_{1}\right) .
\end{aligned}
$$

Comparing with results obtained for unitary case [17], we find that the conditional average Eq. (10) is exactly the same. Thus we cannot tell the symmetry of the system only from the averaged amplitude even if we know the conditional one. To detect the symmetry, we have to examine the variance, where there is a factor of 2 difference between the orthogonal and the unitary case. 
In Fig. 1 and 2, we compare the analytical results of the conditional average and variance with the experimental data from microwave cavities. The experimental curves were obtained by picking points in a wavefunction with the same value and calculating the average wavefunction value a distance $r$ from it on a circle. This quantity was then again averaged over at least 50 wavefunctions after rescaling the wave number to obtain better statistics. Fig. 1 shows the plot of $\left\langle v_{2}\right\rangle_{v_{1}}$ in Eq. (10) with experimental results for $v_{1}=2$ and $v_{1}=7$. Very good agreement is seen for both sets. In fact, agreement is excellent for all values of $v_{1}$ above 1 , below which the noise and errors in the measurement of the wavefunction measurements lead to qualitative differences. In Fig. 2, the comparison of the data to the expression in Eq. (11) was done. Again one sees an excellent agreement with experimental errors of $5 \%$, which is the level of experimental accuracy.

According to Eqs. (10,11), we can say, as in the unitary case [17], that large fluctuations of the wavefunction have some striking structure which is not present for small fluctuations. For $v_{1} \gg 1$ the ratio of the variance to the average square is

$$
\frac{\left\langle\left(\delta v_{2}\right)^{2}\right\rangle_{v_{1}}}{\left\langle v_{2}\right\rangle_{v_{1}}^{2}} \approx 2\left(1-f^{2}\right) \frac{1+2 v_{1} f^{2}}{\left(1+v_{1} f^{2}\right)^{2}}
$$

Therefore at $r \lesssim \xi$, where the "correlation length" $\xi \sim k^{-1} v_{1}^{1 /(d-1)} \gg k^{-1}$, the variance $\left\langle\left(\delta v_{2}\right)^{2}\right\rangle_{v_{1}}$ can be very small in comparison with $\left\langle v_{2}\right\rangle_{v_{1}}^{2}$. It means, that once we know that the wavefunction $|\psi(\mathbf{r})|^{2}$ is equal to $v_{1}$ at $\mathbf{r}_{1}$, it is highly likely to have a value $\left\langle v_{2}\right\rangle_{v_{1}} \sim f^{2} v_{1}$ at $\mathbf{r}_{2}$ for $r \lesssim \xi$. In this sense, the large fluctuation behavior of the wavefunction becomes highly predictable. In contrast, for small fluctuations $v_{2} \ll 1$, we easily see that $\left\langle\left(\delta v_{2}\right)^{2}\right\rangle_{v_{1}} \approx$ $2\left\langle v_{2}\right\rangle_{v_{1}}^{2}$, independent of $v_{1}$. We also find directly from Eq. (5) that fluctuations turn out to be independent, i.e., $P_{v_{1}}\left(v_{2} ; r\right) \approx 1 / \sqrt{v_{2}}$. Although more careful evaluation gives us a correlation length $\xi$ which ensures independent fluctuations for the region $r \gtrsim \xi$, $\xi$ in such an evaluation turns out to be very small, i.e., $\xi \sim k^{-1} \sqrt{v_{1}} \ll k^{-1}$.

These behaviors are qualitatively the same both in systems with or without time-reversal symmetry. Therefore we can say that this is a generic property of chaotic wavefunctions. Also, in the semiclassical description of chaotic systems, periodic and closed orbits are known 
to be associated with large values of the wavefunctions $|\psi(\mathbf{r})|^{2}$ [27,28]. In this respect, our present results may imply there is some structure present in these orbits.

Now let us proceed with the derivation of our main result in Eqs. (5.66). To evaluate the joint distribution $P\left(v_{1}, v_{2} ; r\right)$, we work with its moments,

$$
q_{n m}(r)=V^{n+m}\left\langle\left|\psi_{\epsilon}\left(\mathbf{r}_{1}\right)\right|^{2 n}\left|\psi_{\epsilon}\left(\mathbf{r}_{2}\right)\right|^{2 m}\right\rangle \equiv\left\langle v_{1}^{n} v_{2}^{m}\right\rangle
$$

$q_{n m}$ is known to be closely related to the moments of the exact retarded and advanced Green functions $G_{\gamma}^{R, A}$,

$$
F_{n m}(r ; \gamma)=\frac{i^{n-m}}{(\pi \nu)^{n+m}}\left\langle\left(G_{\gamma}^{R}\left(\mathbf{r}_{1}, \mathbf{r}_{1}\right)\right)^{n}\left(G_{\gamma}^{A}\left(\mathbf{r}_{2}, \mathbf{r}_{2}\right)\right)^{m}\right\rangle
$$

where $\nu$ is the average DOS and

$$
G_{\gamma}^{R, A}\left(\mathbf{r}_{1}, \mathbf{r}_{2}\right)=\sum_{\alpha} \frac{\psi_{\alpha}\left(\mathbf{r}_{1}\right) \psi_{\alpha}^{*}\left(\mathbf{r}_{2}\right)}{\epsilon-\epsilon_{\alpha} \pm i \gamma / 2}
$$

We can obtain $q_{n m}$ in terms of $F_{n m}$ by the relation [25,26]

$$
q_{n m}=\frac{(n-1) !(m-1) !}{2(n+m-2) !} \lim _{\gamma \rightarrow 0}\left(\frac{\gamma}{\Delta}\right)^{n+m-1} F_{n m}
$$

since the leading contribution to $F_{n m}$ for small $\gamma$ comes from the state whose energy $\epsilon_{\alpha}$ coincides with $\epsilon$.

$F_{n m}$ can be evaluated by the supermatrix method. However, since we cannot utilize a simple expression like Eq. (12) of Ref. [17 for the orthogonal case, we are forced to expand $F_{n m}$ directly by the Friedel function $f(r)$ as (see also Ref. [12,21)

$$
F_{n m}(r ; \gamma)=n ! m ! \sum_{q} C_{q}(n, m) f^{2 q}(r)
$$

Using the same notation for the supermatrix elements as in Ref. [19], and defining $\tilde{Q}^{a b} \equiv$ $(-1)^{a} Q^{a b}$ (for $\left.a=1,2\right)$, the coefficient $C_{q}(n, m)$ in Eq. (17) is given in terms of $Q$-supermatrix elements:

$$
C_{q}(n, m)=\sum \prod_{a, b=1}^{2} \frac{\left\langle\left(\tilde{Q}_{34}^{a b}\right)^{k_{a b}}\left(\tilde{Q}_{43}^{a b}\right)^{p_{a b}}\left(\tilde{Q}_{33}^{a b}\right)^{l_{a b}}\right\rangle_{Q}}{\left(1+\delta_{a b}\right)^{k_{a b}+p_{a b}} k_{a b} ! p_{a b} ! l_{a b} !}
$$


where the summation is taken over the all possible combinations of nonnegative integer $k_{a b}$, $p_{a b}, l_{a b}$ which satisfy the condition: $2 q=\sum_{a \neq b}\left(k_{a b}+p_{a b}+l_{a b}\right), m-\sum_{a} l_{1 a}=2 k_{11}+\sum_{a \neq b} k_{a b}=$ $2 p_{11}+\sum_{a \neq b} p_{a b}$, and $n-\sum_{a} l_{a 2}=2 k_{22}+\sum_{a \neq b} k_{a b}=2 p_{22}+\sum_{a \neq b} p_{a b}$. The symbol $\langle\cdots\rangle_{Q}$ denotes an integration over the saddle point manifold, i.e.,

$$
\langle\cdots\rangle_{Q} \equiv \int D Q(\cdots) \exp [-(\pi \gamma / 4 \Delta) \operatorname{Str} \Lambda Q]
$$

where the definitions of $\Lambda$ and Str as well as the structure of the $Q$ matrix are found in 19 .

In principle, averaging $\langle\cdots\rangle_{Q}$ in Eq. (18) can be carried out by using the parameterization of Ref. [19]. However, we found it technically unfeasible to evaluate this expression in such a general form. Fortunately, to get Eq. (16) one need only to know $F_{n m}(\gamma \rightarrow 0)$. The leading contribution in this limit can be extracted by transforming parameters $\lambda_{i}=1+u_{i} \sqrt{\Delta / \gamma}$ for $i=1,2$ in the parameterization given in Ref. [19. By calculating the leading order of small $\gamma$, we find the relation

$$
\tilde{Q}_{c d}^{a b} \tilde{Q}_{c^{\prime} d^{\prime}}^{a^{\prime} b^{\prime}} \simeq \tilde{Q}_{c d^{\prime}}^{a b^{\prime}} \tilde{Q}_{c d^{\prime}}^{a b^{\prime}}
$$

for $a, b=1,2$ and $c, d=3,4$. Substituting Eq. (20) into Eq. (18) and combining with Eq. (16), we finally obtain after integrating over the $Q$ matrix,

$$
q_{n m}(r)=\sum_{q} \frac{(2 n-1) ! !(2 m-1) ! ! f^{2 q}(r)}{2^{n+m-2 q}(n-q) !(m-q) !(2 q) !} .
$$

Reconstructing $P\left(v_{1}, v_{2} ; r\right)$ from the moments $q_{n m}$ completes the derivation of our main result Eqs. (5, 6).

In conclusion we have presented analytical results for universal statistical quantities which characterize the coordinate dependence of chaotic wavefunctions of the system with time-reversal symmetry. Further we have demonstrated excellent agreement between the theoretical results and experimental results of microwave cavities. The spatial correlations demonstrate the long-range Friedel oscillations of wavefunction density and the existence of extended spatial regions of high wavefunction density. 
The authors are grateful to B. L. Altshuler and B. D. Simons for their interest in the work. This work is partially supported through NSF Grant No. DMR 92-04480 and ONR under Grant No. N00014-92-J-1666. 


\section{REFERENCES}

* On leave from A.F. Ioffe Physico-Technical Institute, 194021 St. Petersburg, Russia.

$\dagger \quad$ Present address: NEC Research Institute, 4 Independence Way, Princeton, NJ 08536.

[1] M. Berry, in Chaos and Quantum Physics, edited by M.-J. Giannoni, A. Voros, and J. Zinn-Justin (North-Holland, Amsterdam, 1991), p. 251

[2] F. Haake, Quantum Signatures of Chaos (Springer-Verlag, Berlin, 1991),

[3] B. L. Altshuler in Nanostructures and Mesoscopic Systems, edited by W. P. Kirk and M. A. Reed (Elsevier, Amsterdam, 1991).

[4] E. P. Wigner, Ann. Math. 53, 36 (1951).

[5] F. J. Dyson, J. Math. Phys. 3, 140 (1962).

[6] M. L. Mehta, 'Random Matrices - Revised and Enlarged Second Edition' (Academic Press Inc., San Diego, 1991).

[7] O. Bohigas, in Chaos and Quantum Physics, edited by M.-J. Giannoni, A. Voros, and J. Zinn-Justin (North-Holland, Amsterdam, 1991), p. 87.

[8] E. J. Heller and S. Tomsovic, Physics Today, 46, 38 (1993).

[9] T. A. Brody et al., Rev. Mod. Phys. 53, 385 (1981).

[10] M. V. Berry, in Chaotic Behavior of Deterministic System, edited by G. Iooss, R. H. G. Helleman and R. Stora, (North Holland, Amsterdam, 1983), p. 171.

[11] P. O'Connor, J. Gehlen, and E. J. Heller, Phys. Rev. Lett. 58, 1296 (1987).

[12] V. N. Prigodin, B. L. Altshuler, K. B. Efetov, and S. Iida, Phys. Rev. Lett. 72, 546 (1994).

[13] M. F. Crommie, C. P. Lutz, and D. M. Eigler, Science 262, 218 (1993). 
[14] Y. Hasegawa and P. Avouris, Phys. Rev. Lett. 71, 1071 (1993).

[15] E. Doron and U. Smilansky and A. Frenkel, Phys. Rev. Lett. 65, 3027 (1990).

[16] S. Sridhar, Phys. Rev. Lett. 67, 785 (1991).

[17] V. N. Prigodin, Phys. Rev. Lett. 74, 1566 (1995).

[18] A. Kudrolli, V. Kidambi, and S. Sridhar, Phys. Rev. Lett. (to be published).

[19] K. B. Efetov, Adv. Phys. 32, 53 (1983).

[20] J. J. M. Verbaarschot, H. A. Weidenmüller, and M. R. Zirnbauer, Phys. Rep. 129, 367 (1985).

[21] N. Taniguchi, A. V. Andreev, and B. L. Altshuler, Europhys. Lett. 29, 515 (1995).

[22] V. I. Fal'ko and K. B. Efetov, Phys. Rev. B 50, 11267 (1994).

[23] J. Friedel, Nuovo Cim. Suppl. 2, 287 (1958).

[24] P. A. Lee, A. D. Stone, and H. Fukuyama, Phys. Rev. B 35, 1039 (1987).

[25] F. Wegner, Z. Phys. B 36, 209 (1980).

[26] B. L. Altshuler and V. N. Prigodin, Sov. Phys. JETP 68, 198 (1989).

[27] E. J. Heller, Phys. Rev. Lett. 53, 1515 (1984).

[28] E. B. Bogomolny, Physics D 31, 169 (1988). 


\section{FIGURES}

FIG. 1. The average spatial dependence of chaotic wavefunction squared $\left\langle v_{2}\right\rangle_{v_{1}}=V\left\langle\left|\psi_{\epsilon}\left(\mathbf{r}_{2}\right)\right|^{2}\right\rangle$ whose value $V\left|\psi_{\epsilon}(\mathbf{r})\right|^{2}$ at a point $\mathbf{r}_{1}$ is known to be $v_{1}\left(r=\left|\mathbf{r}_{2}-\mathbf{r}_{1}\right|, V\right.$ is the volume, and $\left.\hbar^{2} k^{2} /(2 m)=\epsilon\right)$. The theoretical prediction Eq. (10) is compared with experiments from the microwave Sinai stadium cavity for $v_{1}=7(\square)$ and $v_{1}=3(\circ)$. Inset: Representative eigenfunction of the chaotic Sinai stadium.

FIG. 2. Comparison of the conditional variance of a wave function $\left\langle\left(\delta v_{2}\right)^{2}\right\rangle_{v_{1}}\left(\delta v_{2}=v_{2}-\left\langle v_{2}\right\rangle_{v_{1}}\right.$ and $v_{2}=V\left|\psi_{\epsilon}\left(\mathbf{r}_{2}\right)\right|^{2}$ ) as a function of the distance from point $\mathbf{r}_{1}$ and a reference value $v_{1}$ $\left(v_{1}=V\left|\psi_{\epsilon}\left(\mathbf{r}_{1}\right)\right|^{2}\right)$ between the theory (Eq. (11)) and the experiment for $v_{1}=7$ (口) and $v_{1}=3(\circ)$. 


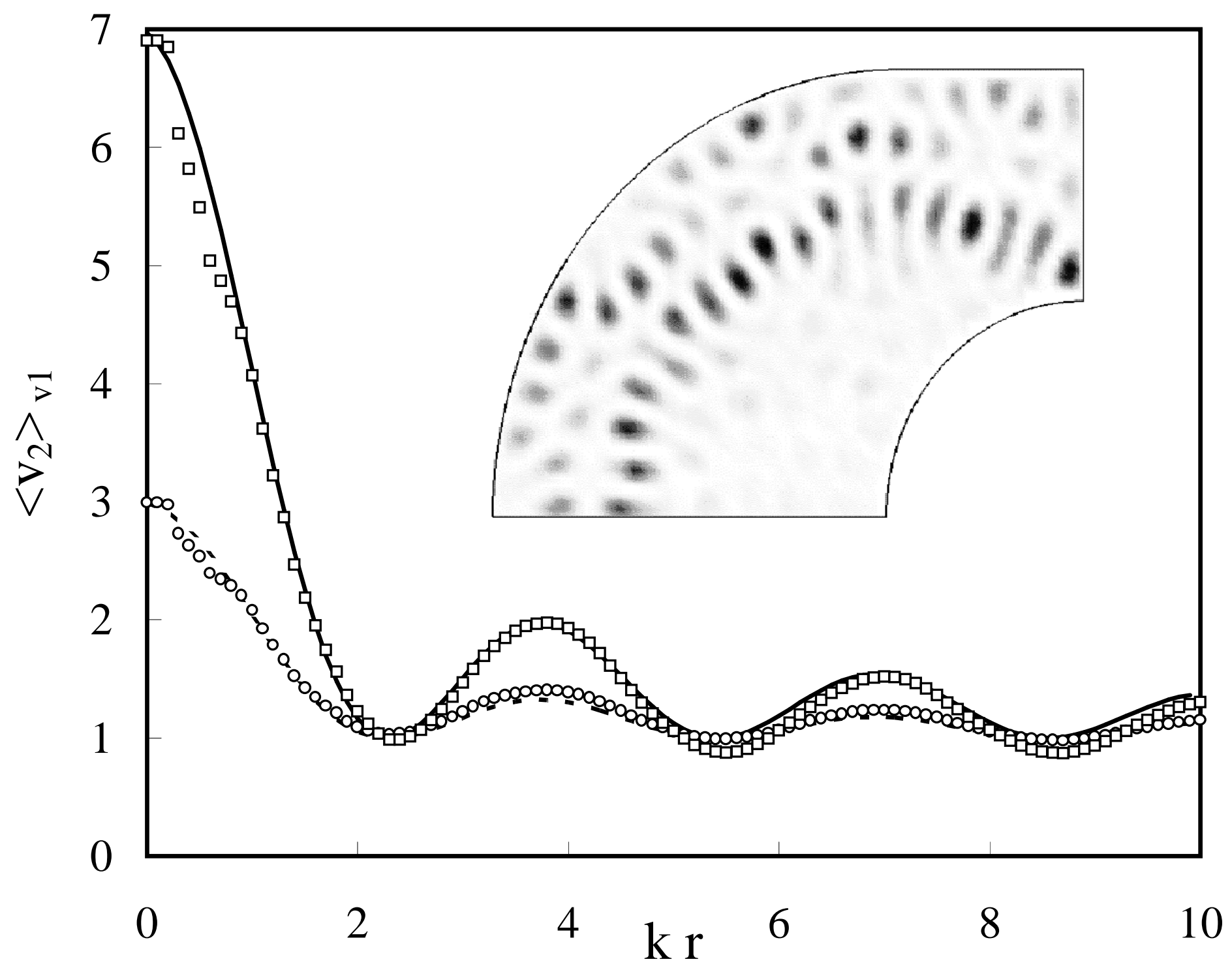




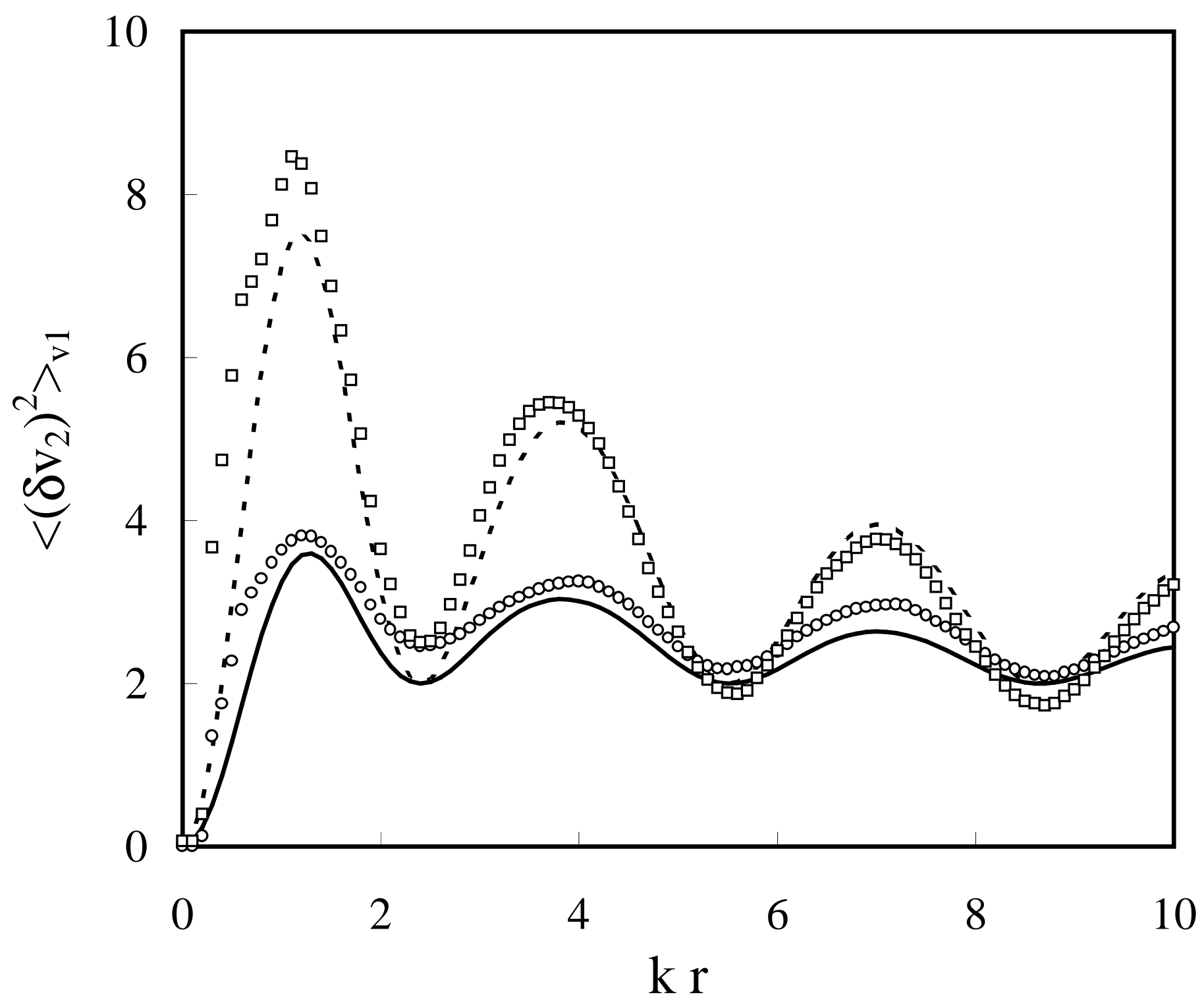

